\title{
A parallel printer port to matrix driver with high current DAC output
}

\author{
WILLIAM B. CUSHMAN \\ Naval Aerospace Medical Research Laboratory, Naval Air Station, Pensacola, Florida
}

\begin{abstract}
The circuit described here takes data from an IBM PC, or a compatible, parallel printer port and expands it to drive a matrix of devices. The matrix size is user definable up to a maximum size of 16 by 16 elements (256 elements), but as drawn is an 8 by 24 matrix (196 elements). Each element in the matrix can be inductive, resistive, or capacitive, with a maximum voltage of about 18 volts, and a total maximum current of 15 amps peak, or 8 amps continuous with adequate heat sinks (dissipating 80 watts maximum per driver device). Several matrix elements may be asserted simultaneously. The voltage applied across the matrix "rails" is controlled by a digitalto-analog converter with 4096 steps (12-bit accuracy).
\end{abstract}

The circuit described in this paper was inspired by the need to address a large number of elements in an array of tactile stimulators, and to operate them with various waveforms and periods. The tactile stimulators were worn around the torso of a subject flying an airplane, with attitude information transmitted to the subject via the pattern of stimulation used. Using this instrumentation, subjects have successfully flown blindfolded. At the Naval Aerospace Medical Research Laboratory, we are interested in reducing the visual workload of a pilot by giving useful information through alternate sensory channels. The use of alternate sensory channels for information input may prove useful for others in high-workload environments or for handicapped individuals.

The experimental paradigm that we are working with requires the entire apparatus to be light and portable, yet flexible enough to allow various parameters to be experimentally defined without rewiring. A portable computer provides the flexibility needed to be used in an aircraft. Most "laptop" computers have very limited provision for "add-on" devices, but almost all have a parallel printer port. The printer port has proven to be an excellent output device for this application. The circuit described here can be configured to fit the needs of the user and can be complemented with a data input channel if needed. Jun (1990) and Dalrymple-Alford (1992) give examples of input circuits, and Eckel (1987), Hogan (1988), and Sargent and Shoemaker (1984) provide additional information about the intricacies of the printer

Trade names of materials and/or products of commercial or nongovernment organizations are cited as needed for precision. These citations do not constitute official endorsement or approval of the use of such commercial materials and/or products. The opinions and conclusions contained in this report are those of the author and do not necessarily reflect the views or the endorsement of the Department of the Navy, Department of Defense, nor the U.S. Government. This research was supported by the Naval Medical Research and Development Command under work unit number 63706N M0096.002-7056. Address correspondence to the author at the Naval Aerospace Medical Research Laboratory, Naval Air Station, Pensacola, FL 32508-5700. port and its programming. The cost of parts to build the circuits described in this paper should be approximately $\$ 150$. The addition of a box, power supply, and so forth, would raise the cost to about $\$ 200$.

\section{Theory of Operation}

The parallel printer port on a PC computer has eight data lines plus several control lines. The circuit described here uses the control lines to multiplex the data lines into a series of latches. Four of the latches are used to develop the signals necessary to address the matrix. Two more of the latches receive the high and low bytes of a digitalto-analog converter (DAC) input word, and a final pair of latches is used to "double buffer" the DAC input word. Double buffering the DAC input word prevents output "glitches" that would occur if the input bits of the 12-bit word were not presented simultaneously. Shortly after the DAC input word is presented to the DAC, the output current reflects this numerical magnitude. The particular DAC specified in Figure 1 has a current output, so conversion must be made to voltage and provision made to provide the power required. This is accomplished with an operational amplifier driving a triple-Darlington power amplifier.

The output of the power amplifier is fed to the inputs of the row-select transistors where it can be directed to the matrix "rails" by asserting the particular row of interest via the latched bit for that particular row. In a similar fashion, a column can be selected that directs that particular column-select transistor to become conductive to ground. When a particular row-select transistor and a corresponding column-select transistor are simultaneously asserted, a path for current exists through these transistors from the DAC power amplifier to ground via whatever element is wired across this particular row and column.

\section{Detailed Description of the Circuit}

In Figure 1, inputs from the printer port of an IBM PC or compatible are used to select rows and columns plus 

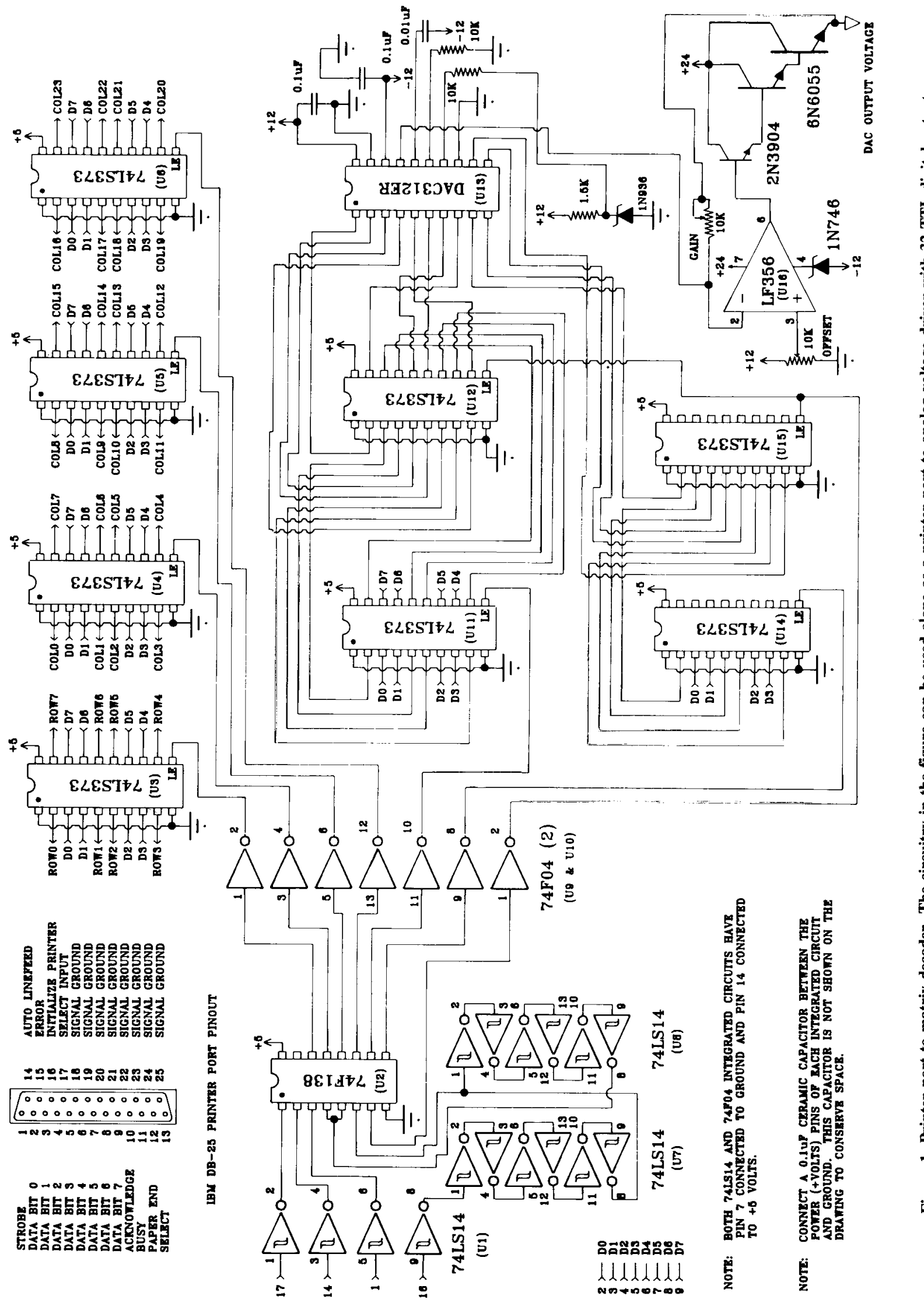

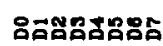

llldlld 蓆 
a DAC output voltage. A data byte from the PC's printer port is presented to the circuit on pins 2 through 9 , with pin 2 the least significant bit (LSB). These pins are asserted by writing the data byte to port $0378 \mathrm{~h}$. Pins 17 , 14 , and 1 are inverted outputs (select input, auto feed, and strobe, respectively) from port 037Ah and make up the address lines for $\mathrm{U} 2$, after being again inverted and buffered by U1. U2 is a three-line to eight-line demultiplexer. Pin 16 is a "strobe" ("initialize printer") from port 037Ah that causes the address lines at $\mathrm{U} 2$ to be "gated" by U2 after a delay of approximately $132 \mathrm{nsec}$ caused by the string of inverters in U7. Even though the signals on pins $17,14,1$, and 16 can be written simultaneously (with the write instruction), it must be assumed that they will not appear at the device in Figure 1 simultaneously, because of varying bit delays on the lines. The 132-nsec delay imposed by U7 allows the signals to settle to a stable level before $U 2$ is enabled by asserting $U 2$ pin 6 . U8 then provides an additional 132-nsec delay before $\mathrm{U} 2$ is disabled by a signal at $\mathrm{U} 2$ pins 4 and 5 . In this fashion, the output of $U 2$ is asserted for approximately $132 \mathrm{nsec}$, and only the output line indicated by the address inputs is asserted. So, for example, the address at U2 pins 3, 2, and 1 could be 0,0 , and 1 , respectively (binary 1), and a 132-nsec pulse would appear at U2 pin 14. This negative-going pulse is then inverted by U9 and used to assert the Latch Enable (LE) input of U3, thus causing the data byte, D0 to D7, to be latched through to ROW0 to ROW7, respectively. The output of this latch then remains until either power fails, or it is programmed again. In a similar fashion, an address of 0,1 , and 0 (binary 2) would cause $U 4$ to latch the data byte through to its output.

A binary 3 at $U 2$ pins 3,2 , and 1 will select U5, and a binary 4 will select U6. The outputs from U3 through U6 form the rows and columns of the matrix after passing through the drivers shown in Figure 2. U11 and U14 are addressed with binary 5 and 6, respectively, and are used to write the low and high bytes for the DAC from the data bits. After both the high and low bytes have been written to U11 and U14, the DAC input word is present at the inputs to U12 and U15. A write to binary 7 then activates the LE of both U12 and U15, thus causing the full 12-bit DAC input word to be presented simultaneously. When a write is made to binary 7 , the data bits D0 through D7 are ignored.

The current output from the DAC (U13) goes to the inverting input of U16, where it is converted to a voltage. The output from U16 drives a 2N3904 transistor in a Darlington configuration with a 2N6055 Darlington power transistor. The emitter of the $2 \mathrm{~N} 6055$ is then connected to a $10 \mathrm{~K}$ trimpot gain control that provides the feedback needed by U16. A second 10K trimpot is used for offset adjustments.

We proceed now to Figure 2 to see how these various signals are used. An inspection of Figure 2 shows that it is highly redundant, so we will simply address the drivers for one row and one column and that should suffice for the whole. Assume that data (binary 2) have been latched into U3 of Figure 1 so that pin 5 is asserted high. Pin 5 of U3 asserts ROW1. If U5 has been latched with the same data (such that U5 pin 5 is high), pin 5 of U5 asserts COL9. Also assume that a pair of data bytes has been loaded and latched through to the DAC so that some voltage is present at the DAC output voltage. Looking now at Figure 2, the high voltage at ROW1 will pass through a $10 \mathrm{~K}$ resistor to turn on a $2 \mathrm{~N} 3904$ transistor. This transistor has a $220-\Omega$ resistor connected to its collector, which is connected to the cathode of a lightemitting diode (LED) in a $6 \mathrm{~N} 139$ optical coupler. The anode of the LED is connected to $+5 \mathrm{~V}$. The LED will be turned on, and its light output will bias a transistor in the optical coupler "on." This optical coupler transistor is in a Darlington configuration with a TIP102 Darlington transistor that has the DAC output voltage connected to its collector, and the rail for ROW1 connected to its emitter. Because this TIP102 is biased to the "on" state, the DAC output voltage is connected to the ROW 1 rail. The usual use for an optical coupler is to provide electrical isolation by causing the signal to be transmitted over a "light" link, but in this case the optical coupler is used to provide "level shifting." That is, no matter what the DAC output voltage, if it is sufficiently positive to turn anything on, the operation of the circuit is the same.

The "row rails" provide a positive voltage (the DAC output voltage minus the voltage drop across the TIP102) to all the attached devices, but none of them can operate until a "ground" is also provided. This is accomplished with the column circuits. Assuming that COL9 is asserted, a positive voltage from COL9 will pass through a $10 \mathrm{~K}$ resistor to bias a TIP102 Darlington transistor to the "on" state, thus causing the associated column rail to be grounded (plus the voltage drop across the TIP102). If a device, say a light or a voice-coil type vibrator, is connected between the ROW1 rail and the COL 9 rail, current will flow through it. Note that a diode is in series with the device being driven. This diode prevents "cross-talk" between devices by limiting current flow to one direction. Note also that both the column and the row rails have reverse-biased diode pairs at the ends, connected to $+24 \mathrm{~V}$ and to ground. These diode pairs protect the circuitry from inductive kickback if inductors are being driven.

Obviously more than one column rail could be asserted with a particular row, or vice versa, to turn "on" more than one device at a time. However, if multiple rows and multiple columns are to be asserted simultaneously, care must be taken to avoid addressing more elements in the matrix than intended. A safe approach is to limit the software to address one row at a time and as many columns as wanted, and then multiplex the rows as needed. This is precisely why I have chosen an $8 \times 24$ matrix, so that the multiplexing time is not too long. One must exercise care in determining the amount of current being drawn, because all of the current for the entire matrix passes through the DAC output 2N6055. This transistor should have a heat sink if no others do! 


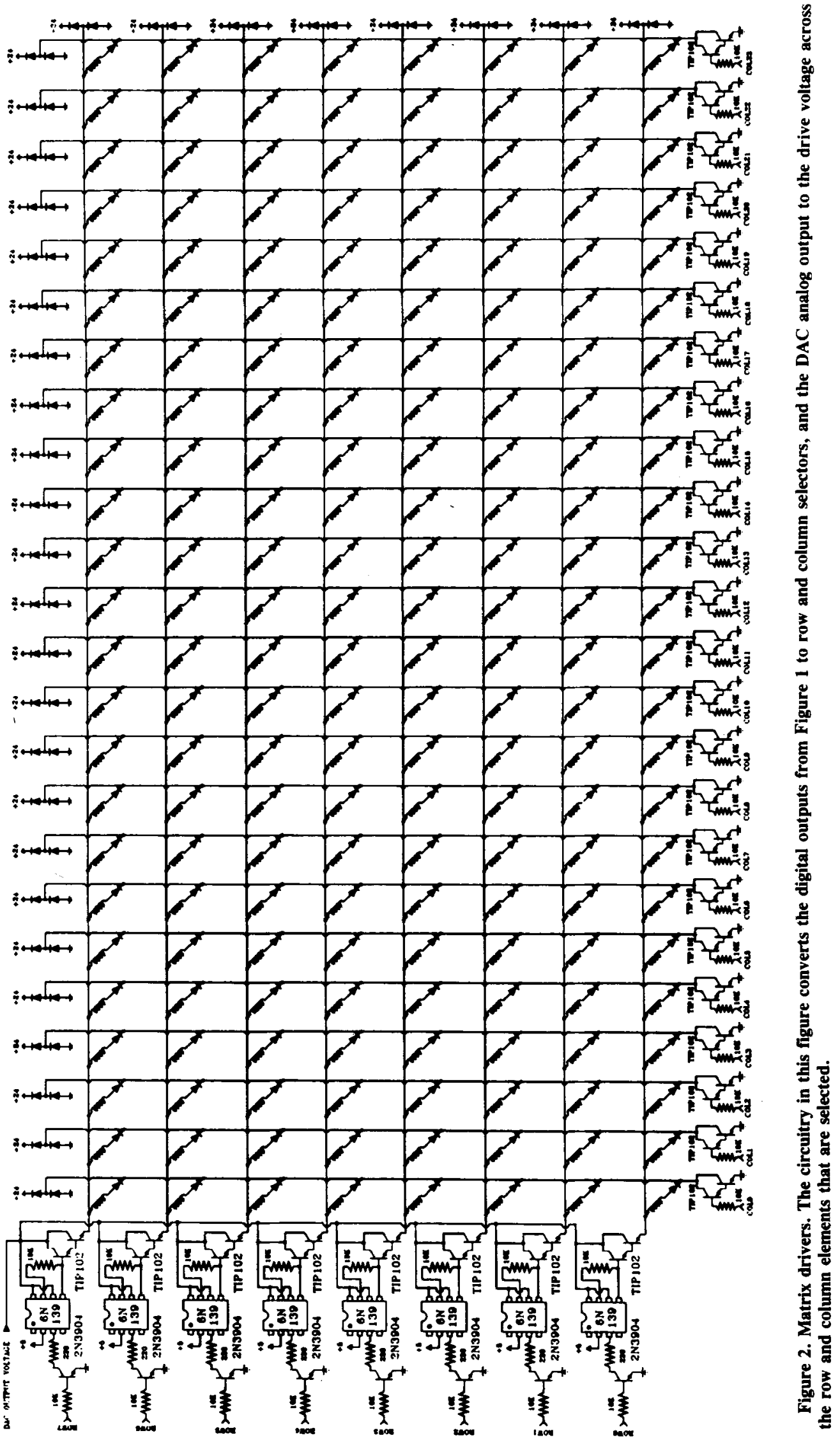




\section{Programming the Device}

Experience with a Zenith Z-248 computer (an 80286 processor running at $8 \mathrm{MHz}$ ) has indicated that output port "writes" average $38 \mu \mathrm{sec}$ each. If a particular element in the array has been selected and a series of DAC outputs is desired, then five "writes" are required for each output. Write the low byte, latch it, write the high byte, latch it, then assert the double latch, for a total time of about $190 \mathrm{msec}$. A common waveform to output is the sine, and a 32-level sine wave looks acceptable after integration in an inductor. Given 32 "outs" per cycle, the maximum frequency would be

$$
\mathrm{Hz}=\frac{1}{32 \times 0.000190},
$$

or more than $164 \mathrm{~Hz}$. Of course, this frequency doubles if 16 levels are acceptable.

Programming is accomplished by first writing the data byte to port $0378 \mathrm{~h}$. If the data byte is for selecting rows or columns, the appropriate bits must be set. Next, the appropriate latch must be asserted. Pin 17 corresponds to the A0 address of the demultiplexer (U2) and is inverted on output to port $037 \mathrm{Ah}$, and again inverted before being presented to U2. This double inversion cancels, so no inversion is required when programming. Pins 14 and 1 are also double-inverted, but not pin 16. However, pin 16 is used as the "strobe," and a zero in that bit location (as written) will be inverted by U1, producing the desired

Table 1

Hex Values to Write to Port 037A and Their Function

\begin{tabular}{|c|c|c|c|c|c|c|}
\hline \multirow[b]{2}{*}{ Latch } & \multirow{2}{*}{$\begin{array}{c}\text { Hex } \\
\text { Value }\end{array}$} & \multicolumn{4}{|c|}{ Pin } & \multirow[b]{2}{*}{ Purpose } \\
\hline & & 17 & 16 & 14 & 01 & \\
\hline U3 & 08 & 1 & 0 & 0 & 0 & Select rows \\
\hline U4 & 02 & 0 & 0 & 1 & 0 & Select columns 0-7 \\
\hline U5 & $0 A$ & 1 & 0 & 1 & 0 & Select columns 8-15 \\
\hline U6 & 01 & 0 & 0 & 0 & 1 & Select columns $16-23$ \\
\hline UII & 09 & 1 & 0 & 0 & 1 & Load low DAC byte \\
\hline U14 & 03 & 0 & 0 & 1 & 1 & Load high DAC byte \\
\hline $\mathrm{U} 12$ and U15 & $\mathrm{OB}$ & 1 & 0 & 1 & 1 & Double buffer \\
\hline
\end{tabular}

output. Table 1 lists the hexadecimal values that must be output to port 037 Ah to address the various latches.

\section{Calibration of Output Levels}

Once the hardware has been assembled and tested, it is necessary to adjust the gain and offset controls indicated in Figure 1 to the voltage levels required. This is most easily accomplished by outputting the values for a sine or triangle wave to the device and observing the waveform with an oscilloscope at the matrix element being driven while adjusting the gain and offset. If the voltage exceeds the positive or negative rails, "clipping" will be obvious and adjustment can be made to compensate. For maximum range, the gain and offset should be adjusted until "clipping" is just noticed, then "backed off" slightly. WARNING: most oscilloscopes carry the receptacle ground from the wall socket through to the probe ground! This means that there is a very real danger of a serious "ground loop" with potential to damage one's equipment. To avoid this problem, either use the oscilloscope in differential mode, making sure the probe ground is never in contact with the circuit being tested; or electrically isolate the oscilloscope with an isolation transformer; or use an isolated computer (such as a "laptop" type) on battery power only, and an isolated power supply for the device described in this paper.

\section{REFERENCES}

DAlrymple-Alford, E. C. (1992). Response-key input via the IBM PC/XT/AT's parallel printer port. Behavior Research Methods, Instruments, \& Computers, 24, 78-79.

ECKEL, B. (1987). A programmer's guide to the parallel port. Turbo Technix, 1, 74-79.

Hogan, T. (1988). The programmer's PC sourcebook. Redmond, WA: Microsoft Press.

Jun, L. (1990). Connect ADC to PC's I/O port. Electronic Design, 38(19), 109.

Sargent, M. \& Shoemaker, R. L. (1984). The IBM Personal Computer from the inside out. Reading, MA: Addison-Wesley.

(Manuscript received January 21, 1992; revision accepted for publication August 18, 1992.) 